\title{
Mutual interferences and design principles for mechatronic devices in magnetic resonance imaging
}

\section{Journal Article}

Author(s):

Yu, Ningbo; Gassert, Roger (D); Riener, Robert

Publication date:

2010-07

Permanent link:

https://doi.org/10.3929/ethz-b-000022225

Rights / license:

In Copyright - Non-Commercial Use Permitted

Originally published in:

International Journal of Computer Assisted Radiology and Surgery 6(4), https://doi.org/10.1007/s11548-010-0528-2 


\title{
Mutual interferences and design principles for mechatronic devices in magnetic resonance imaging
}

\author{
Ningbo Yu • Roger Gassert - Robert Riener
}

Received: 18 March 2010 / Accepted: 16 August 2010 / Published online: 2 September 2010

(c) CARS 2010

\begin{abstract}
Purpose Robotic and mechatronic devices that work compatibly with magnetic resonance imaging (MRI) are applied in diagnostic MRI, image-guided surgery, neurorehabilitation and neuroscience. MRI-compatible mechatronic systems must address the challenges imposed by the scanner's electromagnetic fields. We have developed objective quantitative evaluation criteria for device characteristics needed to formulate design guidelines that ensure MRI-compatibility based on safety, device functionality and image quality.

Methods The mutual interferences between an MRI system and mechatronic devices working in its vicinity are modeled and tested. For each interference, the involved components are listed, and a numerical measure for "MRI-compatibility" is proposed. These interferences are categorized into an MRI-compatibility matrix, with each element representing possible interactions between one part of the mechatronic system and one component of the electromagnetic fields. Based on this formulation, design principles for MRI-compatible mechatronic systems are proposed. Furthermore, test methods are developed to examine whether a mechatronic device indeed works without interferences within an MRI system. Finally, the proposed MRI-compatibility criteria and
\end{abstract}

N. Yu $(\varangle) \cdot R$. Riener

Sensory-Motor Systems Lab, ETH Zurich, Zurich, Switzerland

e-mail: yu@mavt.ethz.ch

R. Gassert

Rehabilitation Engineering Lab, ETH Zurich, Zurich, Switzerland

e-mail: gassertr@ethz.ch

R. Riener

Medical Faculty, Balgrist University Hospital,

University of Zurich, Zurich, Switzerland

e-mail: riener@mavt.ethz.ch design guidelines have been applied to an actual design process that has been validated by the test procedures.

Results Objective and quantitative MRI-compatibility measures for mechatronic and robotic devices have been established. Applying the proposed design principles, potential problems in safety, device functionality and image quality can be considered in the design phase to ensure that the mechatronic system will fulfill the MRI-compatibility criteria.

Conclusion New guidelines and test procedures for MRI instrument compatibility provide a rational basis for design and evaluation of mechatronic devices in various MRI applications. Designers can apply these criteria and use the tests, so that MRI-compatibility results can accrue to build an experiential database.

Keywords MRI-compatibility · Image quality · Electromagnetic interference · Design principles . Mechatronics $\cdot$ Robotics

\section{Introduction}

Motivation

Magnetic resonance imaging (MRI or MR imaging) has become a standard medical imaging modality to visualize body structure using high magnetic fields, and functional MRI (fMRI) measures signal changes in the brain resulting from neural activation. Robotic systems and mechatronic devices that can work compatibly with MRI procedures, find wide applications in various clinical and research areas [1-3]:

- MRI-compatible robots can precisely measure and control position and forces/torques and therefore are able to 
generate guiding force fields or other haptic cues to assist the surgeon during interventional MRI. For example, an MRI-compatible robot can precisely place the needle to the specific position during biopsies.

- As stimulators in somatosensory function mapping experiments, robots can precisely and repetitively exert the designed stimuli to multiple subjects. The onset and duration of the stimuli can also be well controlled. As a result, the systematic error of the study is reduced, the sensitivity of brain activation mapping is increased, and the statistical power of data analysis is elevated.

- An MRI-compatible robot allows well-controlled and reproducible sensorimotor tasks, while the neural underpinnings of this motor interactions can be investigated with fMRI. Application of the robotic system to healthy subjects and patients with neurological disorders such as spinal cord injury (SCI) and stroke would allow reproducible recordings of brain responses during standardized upper/lower extremity movements and thus, provide insights into motor control, cortical reorganization after neurological lesion, produce a better understanding of therapy-induced recovery, and eventually help to derive more efficient rehabilitation strategies.

- Development of MRI-compatible implants will remove the barriers hindering the implants' host patients from receiving MRI examinations.

However, the strong static and dynamic electromagnetic fields bring challenging obstacles to the development of mechatronic devices for use in MRI environments. Issues of safety, proper device functioning as well as image quality have to be addressed before devices are introduced into the MRI environment.

To deal with the technical challenges imposed by the electromagnetic fields, design criteria have been proposed to guide device developers [4]. The applicability of different materials [5], sensing and actuation methods were investigated $[6,7]$. To ensure the devices are safe and work compatibly with MRI procedures, many test methods and standards were formulated [8-14].

The field of MRI-compatible mechatronic devices and their use for various MRI/fMRI applications is rapidly evolving, and there are a growing number of devices entering the field. Nevertheless, it is quite common that only primitive compatibility tests are performed before bringing the devices into an MRI environment, which cannot fully certify the compatibility of the devices with MRI/fMRI procedures and may have negative effects on the application. This can be explained by the fact that MRI-compatible robotics is a very interdisciplinary field, requiring interaction of engineers, MR physicists, medical doctors, neuroscientists, etc., making communication and profound knowledge in all the fields impossible. Further, not every MR center has a local
MR physics group which can carry out detailed compatibility tests. Finally, every MR suite is different, ranging from dedicated penetration panels with integrated signal and power line filters, to setups where the door to the scanner room is left open to guide electric cables into the room. Device development is still in an iterative "build-and-test" loop, and a clear design process that ensures compatibility of the device with MRI/fMRI procedures is not available so far.

MRI-compatible: a qualitative description

In 1997, the terms "MR Safe" and "MR Compatible" were defined by the U.S. FDA [8]. They also appeared in a draft document by GE Medical Systems [9] and in the ASTM standards [15]. In 2005, ASTM redefined "MR Safe" and introduced "MR Conditional" and "MR Unsafe" as active terms, while "MR Compatible" was not redefined and is not used in current standards [10]. The U.S. FDA followed in 2008 [14]. All the three terms are about safety. Neither image artifact nor proper functioning of an introduced device is covered, which are crucial for mechatronic systems. Robotic systems and mechatronic devices can rarely fulfill the requirements for the "MR Safe" label and mostly fall into the category of "MR Conditional" [13].

"MRI-compatible" is a convenient term that embodies the combination of safety, proper functioning of the device and MRI image quality. Within the scope of this paper, "MRIcompatible" indicates that the device has been demonstrated

(1) not to pose any known hazards,

(2) not to have its intended functions deteriorated by the MRI system,

(3) not to significantly affect the quality of the diagnostic information,

in the context of a defined application, imaging sequence and placement within a specified MRI environment.

This description is based on the new definition of "MR Safe", the obsolete definition of "MR Compatible", and the definition in [16]. It gives a qualitative criterion on whether mechatronic devices are able to work compatibly with MRI procedures.

\section{Content of this paper}

First, we formulate the possible mutual interferences between an MRI system and mechatronic devices and give a quantitative measure for each interference. Based on that, we propose design principles for MRI-compatible devices, so that potential problems of safety, device functioning and image quality can be considered and prevented in the design phase. Then, we present test methods to evaluate whether a mechatronic device fulfills the MRI-compatibility requirements. 
Table 1 Technical data of a Philips 3.0T scanner $([17,18])$

\begin{tabular}{llc}
\hline$\vec{B}_{0}(\vec{r})$ & Nominal amplitude $(\mathrm{T})^{\dagger}$ & 3.0 \\
& Homogeneity $(\mathrm{ppm})^{\ddagger}$ & 0.6 \\
& Stability per hour $(\mathrm{ppm})$ & 0.1 \\
& Maximum $|\vec{\nabla}| \vec{B}_{0}||(\mathrm{T} / \mathrm{m})$ & 17.0 \\
& Maximum $\left|\vec{B}_{0}\right| \cdot|\vec{\nabla}| \vec{B}_{0}||\left(\mathrm{T}^{2} / \mathrm{m}\right)$ & 48.0 \\
$\vec{G}(t)$ & Nominal amplitude $(\mathrm{mT} / \mathrm{m})$ & 40 \\
& Maximum slew rate $(\mathrm{mT} / \mathrm{m} / \mathrm{ms})$ & 120 \\
& Minimum rise time $(\mathrm{ms})$ & 0.33 \\
$\vec{B}_{1}(t)$ (head coil) & Nominal amplitude $(\mu \mathrm{T})$ & 20.0 \\
& Nominal frequency $(\mathrm{MHz})$ & 127.73 \\
& Maximum frequency offset $(\mathrm{KHz})$ & 415.0 \\
& Peak power $(\mathrm{kW})$ & 25 \\
\hline
\end{tabular}

${ }^{\dagger}$ For comparison, the earth's magnetic field is $30-60 \mu \mathrm{T}$

* Parts per million, $10^{-6}$

An example is provided to illustrate the proposed design guidelines.

\section{Mutual interferences between an MRI system and mechatronic devices}

Magnetic resonance imaging

In a classical macroscopic view, the magnetic field at a small voxel $\vec{r}$ of the imaging volume in the three-dimensional space consists of a homogeneous static field $\vec{B}_{0}(\vec{r})$, a pulsed gradient magnetic field $\vec{G}(t)$ and the pulsed radio frequency field $\vec{B}_{1}(t)$. The three magnetic fields are usually generated by a strong magnet together with shim coils, gradient coils and radio frequency transmission coils. Theoretically, the field in the imaging area is the superposition of all three fields,

$\vec{B}(t, \vec{r})=\left[\begin{array}{c}0 \\ 0 \\ B_{0}\end{array}\right]+\left[\begin{array}{c}0 \\ 0 \\ \vec{G}(t) \cdot \vec{r}\end{array}\right]+B_{1}(t)\left[\begin{array}{c}\cos (\omega t) \\ \sin (\omega t) \\ 0\end{array}\right]$

where $\vec{r}=\left[\begin{array}{c}x \\ y \\ z\end{array}\right], \vec{G}(t)=\left[\begin{array}{l}G_{x}(t) \\ G_{y}(t) \\ G_{z}(t)\end{array}\right]$.

A strong static field (Table 1) is necessary for good signal quality and high signal-to-noise-ratio (SNR). The field strength determines the resonance frequency, also called Larmor frequency, $\omega$ in Eq. (1)

$\omega(\vec{r})=\gamma B=\gamma\left(B_{0}+\vec{G} \cdot \vec{r}\right)$

Therefore, the voxel position $\vec{r}$ is correlated with the local resonance frequency, at which protons absorb and emit energy. The gradient field selects a specific slice and encodes the location information into the frequency and phase of free

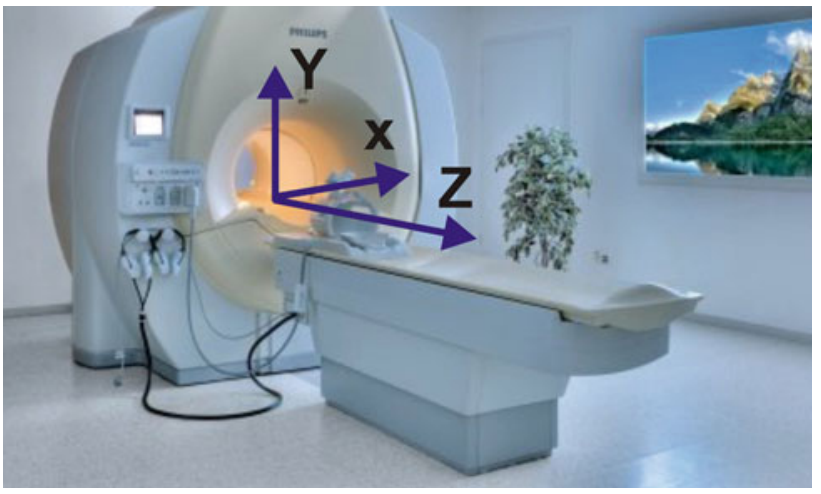

Fig. 1 An MRI scanner in the MR center, ETH and University Zurich

induction decay signals collected by the MRI system. Radio frequency pulses provide energy to the spins and excite them. The static field and the radio frequency field should both be homogeneous over the total volume of interest and orthogonal to each other. Depending on the sequence, images can be weighted by proton concentration, longitudinal relaxation time $T_{1}$, transverse relaxation time $T_{2}$ or their combinations.

The MRI environment

The actual magnetic field differs from the ideal model given by Eq. (1). When the longitudinal gradient $G_{z}$ is simultaneously activated with a transversal gradient $G_{x}$ or $G_{y}$, concomitant gradient cross-terms arise [19]. Scanner system imperfections and external interferences result in field distortions. We denote the overall distortion field that adds to $\vec{B}(t, \vec{r})$ as $\triangle \vec{B}(t, \vec{r})$, which may include static and/or dynamic components. (Fig. 1)

A simplified Biot-Savart model shows that the induced magnetic field along the scanner axial line is inversely proportional to the third power of the distance to the center of the coil

$\vec{B}_{0}\left(\left[\begin{array}{l}0 \\ 0 \\ z\end{array}\right]\right)=\left[\begin{array}{c}0 \\ 0 \\ k / z^{3}\end{array}\right]$

With a set of measured pairs of the magnetic field amplitude and corresponding location, the coefficient $k$ can be calculated by minimizing the root mean square error. Thus, the spatial gradient of the static field along the $z$-axis is $-3 B_{0}(z) / z$. Due to active shielding of the scanner system, $\vec{B}_{0}(\vec{r})$ normally decreases more rapidly than shown above outside the imaging area (Fig. 2, adapted from [20]).

The Maxwell-Faraday equation gives the induced electric field in the MRI environment:

$\vec{\nabla} \times \vec{E}(t)=-\frac{\partial}{\partial t} \vec{B}(t, \vec{r})$ 


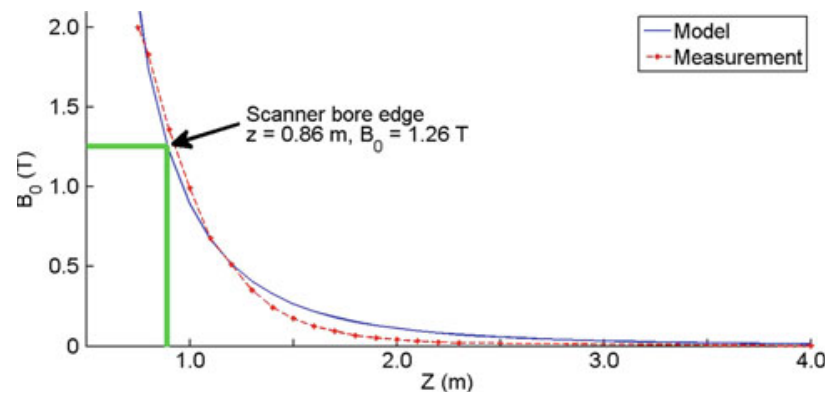

Fig. 2 The magnetic field decreases rapidly with distance from the magnet (adapted from [20]). Dashed red measured curve. Solid blue model curve based on Eq. (3)

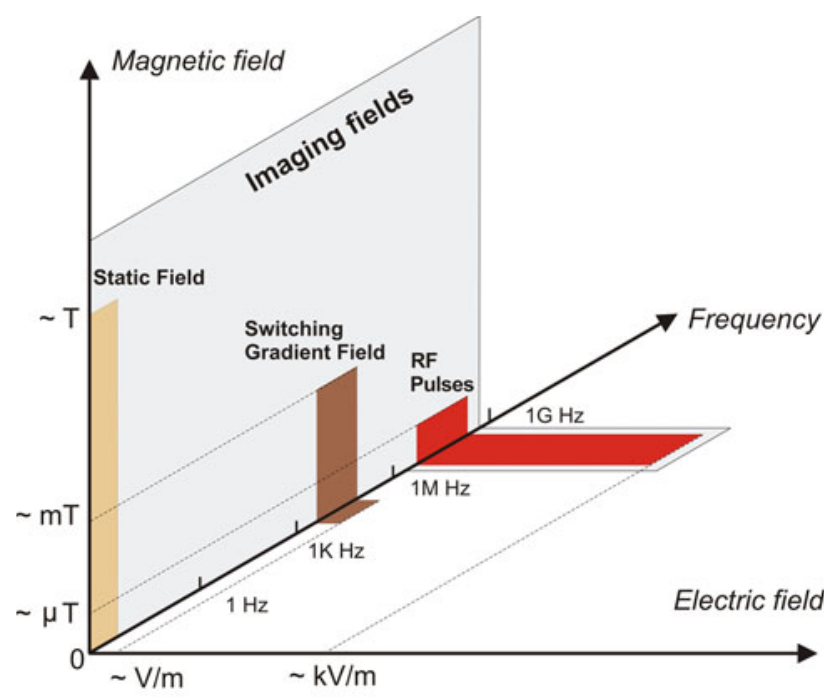

Fig. 3 Electromagnetic fields in the MRI environment: amplitude and frequency distribution (adapted from [21])

According to Eq. (1),

$$
\frac{\partial}{\partial t} \vec{B}(t, \vec{r})=\left[\begin{array}{c}
\frac{\partial}{\partial t} B_{1}(t) \cdot \cos (\omega t)-B_{1}(t) \cdot \omega \sin (\omega t) \\
\frac{\partial}{\partial t} B_{1}(t) \cdot \sin (\omega t)+B_{1}(t) \cdot \omega \cos (\omega t) \\
\frac{\partial}{\partial t} \vec{G}(t) \cdot \vec{r}
\end{array}\right]
$$

The switching time for $B_{1}(t)$ is a few microseconds. For the gradient field, its slew rate ranges from 20 to $200 \mathrm{mT} / \mathrm{m} / \mathrm{ms}$, and the switching frequency is typically in the order of $\mathrm{kHz}$. In contrast, $\omega$ is tens of $\mathrm{MHz}$ or higher (Table 1; Fig. 3). Consequently,

$\frac{\partial}{\partial t} B_{1}(t) \ll B_{1}(t) \cdot \omega, \quad \frac{\partial}{\partial t} \vec{G}(t) \cdot \vec{r} \ll B_{1}(t) \cdot \omega$

Therefore,

$\frac{\partial}{\partial t} \vec{B}(t, \vec{r}) \simeq\left[\begin{array}{c}-B_{1}(t) \cdot \omega \sin (\omega t) \\ B_{1}(t) \cdot \omega \cos (\omega t) \\ 0\end{array}\right]$ and

$\vec{E}(t) \simeq\left[\begin{array}{c}0 \\ 0 \\ B_{1}(t) \omega(x \cos \omega t+y \sin \omega t)\end{array}\right]$

The MRI process is sensitive to magnetic field inhomogeneities and RF noise. Field inhomogeneities mixed with the gradient field results in localization error. Therefore, $\vec{B}_{0}$ must be highly homogeneous and temporally stable in the imaging area (Table 1). RF noise can influence the resonance process or be mistaken as signal by the MRI system, both of which bring incorrect image information. For example, increased background noise will lead to reduced signal-noise ratio (SNR).

An MRI system interacts with mechatronic devices

Interactions between an MRI system and mechatronic components were discussed in $[4,6]$. Magnetic fields in an MRI environment act on a device in multiple ways. $\vec{B}_{0}$ and its spatial gradient exerts force/torque on magnetic materials and current carrying cables. It also disturbs electromagnetically active components. Currents can be induced in conductive materials or closed-loop structures, if they move or the magnetic field varies or both. The induced currents produce heat, generate local magnetic fields and yield noise in electronic signals.

On the other hand, the imaging fields may also be distorted by the mechatronic device. Material magnetization and magnetic fields generated by electric currents lead to spatial localization errors and signal void in the imaging procedure. Electromagnetic noise in the RF bandwidth interferes with MRI signals. Low-frequency noise may be mistaken for neural activity related MRI signal changes in fMRI applications.

\section{Magnetic force}

The high static magnetic field and its spatial gradient exert magnetic force on current carrying wires or components that can be magnetized.

The Lorenz force on a current carrying wire is $\vec{F}_{M}=$ $I \vec{B} \times \vec{L}$. For non-homogeneous field outside the imaging area, the resulting force can be calculated by integration

$\vec{F}_{M}=\int I \vec{B}(\vec{L}) \times d \vec{L}$

When a material of magnetic susceptibility $\chi$ and volume $V$ is placed within a magnetic field $\vec{B}$, the induced magnetization is:

$\vec{M}= \begin{cases}\frac{1}{\mu_{0}} \chi \vec{B} V, & \text { if } \frac{1}{\mu_{0}}|\chi| B V \leq\left|\vec{M}_{s}\right| \\ \vec{M}_{s}, & \text { if } \frac{1}{\mu_{0}}|\chi| B V>\left|\vec{M}_{s}\right|\end{cases}$ 
where $\mu_{0}$ is the magnetic constant $4 \pi \times 10^{-7} \mathrm{~N} / \mathrm{A}^{2}$, and $\vec{M}_{S}$ is the magnetic saturation. The magnetic force is

$$
\vec{F}_{M}=\vec{\nabla}(\vec{M} \cdot \vec{B})
$$

For a homogeneous field $\vec{B}, \vec{M}$ is constant and so is $\vec{M} \cdot \vec{B}$. Therefore, $\vec{\nabla}(\vec{M} \cdot \vec{B})=0$, i.e., the force is zero. In the saturation case, the magnetic force is maximal where $\vec{B}$ shows its maximal gradient. Below the saturation level, the magnetic force is maximal where the product of the field strength $|\vec{B}|$ and its gradient $|\vec{\nabla}| \vec{B}||$ are maximal. Normally, these two locations coincide at the edge of the imaging area inside the scanner bore [17], which is also suggested by the model in Eq. (3).

To compare the magnetically induced force with the gravity force $F_{g}=m g$, we introduce a measure

$$
\beta_{F} \triangleq \max _{\substack{\text { location, } \\ \text { orientation }}} \frac{\left|\vec{F}_{M}\right|}{F_{g}}
$$

When $\beta_{F}<1$, the component is accelerated faster toward the ground than to the magnet. The other two fields show far smaller amplitudes than $\vec{B}_{0}(\vec{r})$, and their influence on the magnetic force can be neglected.

\section{Magnetic torque}

In the strong magnetic field, a current loop or a component with magnetization $\vec{M}$ experiences magnetically induced torque. The magnetization $\vec{M}$ of components is already given by Eq. (8). For a current loop,

$\vec{M}=\int \vec{A}(I) d I$

where $\vec{A}$ is the enclosed area of the loop. The torque can be computed by

$\vec{\tau}_{M}=\vec{M} \times \vec{B}$

This torque tries to align $\vec{M}$ and $\vec{B}$, thus minimizing the potential energy $-\vec{M} \cdot \vec{B}$.

The magnetically induced torque can be compared with the gravitational torque. We denote the measure

$\beta_{\tau} \triangleq \max _{\substack{\text { location, } \\ \text { orientation }}} \frac{\left|\vec{\tau}_{M}\right|}{\tau_{g}}$

where $\tau_{g}$ is the upper bound of the gravity torque, given by the product of the component's weight and its longest dimension.

Since the other two fields show far lower amplitudes than the static field $\vec{B}(\vec{r})$, their contribution to the magnetic torque can be neglected.

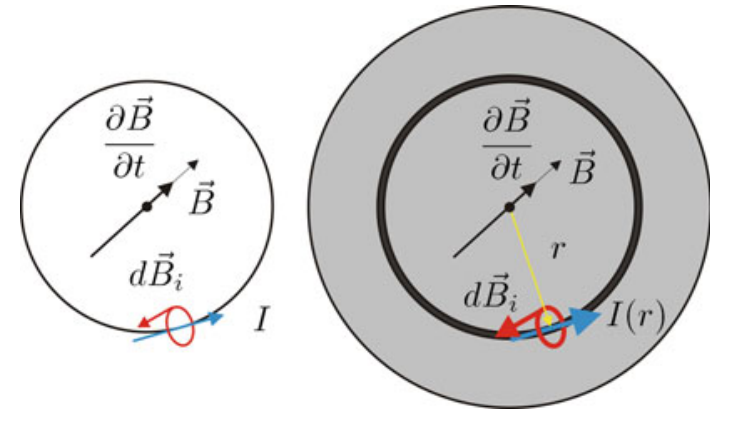

Fig. 4 A magnetic field variation induces a current in a conductive loop (left) and a conductive plate (right). The current generates a local magnetic field to counteract the field variation

\section{Induced current}

A conductive loop or component induces electromotive force and current when the magnetic flux through the loop or component varies. Eddy currents in metallic parts are a source of considerable torsional moment, which may exceed the torque generated by gravity [22].

The induced electromotive force is

$U_{i}=-\frac{\partial \phi}{\partial t}=-\frac{\partial(\vec{B} \cdot \vec{A})}{\partial t}$

where $\sigma$ is the conductance of the loop, $\phi$ is the magnetic flux through the loop. With the loop conductance $\sigma$, the induced current is

$I_{i}=\sigma U_{i}$

If the loop remains in place, we obtain

$I_{i}=-\sigma \int \frac{\partial \vec{B}(A)}{\partial t} \cdot d \vec{A}$

where $\vec{B}(A)$ is the magnetic field through area $A$. In this case, current induction results from the dynamic magnetic fields $\vec{G}(t)$ and $\vec{B}_{1}(t)$.

If one part of the loop is moving, we then obtain

$I_{i}=-\sigma\left(\int \frac{\partial \vec{B}(A)}{\partial t} \cdot d \vec{A}+\vec{B} \cdot \frac{\partial \vec{A}}{\partial t}\right)$

The induced current generates a local magnetic field to counteract the change of magnetic flux $\phi$ (Fig. 4).

However, the calculation of eddy currents, the induced current in conductive components resulting from movement through the field or from radio frequency pulses, is not straightforward. Camacho et al. performed a detailed quantitative analysis of RF-induced eddy currents and related image artifacts using a circular copper loop [23]. A conductive plate (Fig. 4) may be treated as many concentric conductive loops. General eddy current problems cannot be solved analytically or be approximated explicitly, thus requiring advanced 
numerical methods, simulation programs or clear and easyto-use compatibility testing protocols.

\section{Heating}

RF waves may resonate along conducting wires and build up strong electrical energy [24-27], and conductive components and loops can heat up due to induced currents [28].

Electromagnetic waves travel along conducting wires. When the wire length $L$ is multiples of the half wavelength $\lambda_{E M}$, the electromagnetic waves resonate and the wire heats up. The wavelength is calculated by

$\lambda_{E M}=\frac{c}{\sqrt{\epsilon_{r} f}}$

where $c$ is the speed of light $\left(3 \times 10^{8} \mathrm{~m} / \mathrm{s}\right), \epsilon_{r}$ is the relative dielectric constant of the medium surrounding the wire, and $f$ is determined by the field strength as Eq. (2) shows. For water in a $3 \mathrm{~T}$ scanner, we obtain

$f=127.73 \mathrm{MHz}, \quad \epsilon_{r}=81, \quad$ and thus $\lambda_{E M}=0.26 \mathrm{~m}$

Heating occurs to a wire into the human body when the wire length $L$ between the wire tip and the wire entry point into the body is multiples of the half wavelength $\lambda_{E M}$. The energy absorption is significantly reduced by a factor of $\left[L /\left(\lambda_{E M} / 2\right)\right]^{3}$ if $L<\lambda_{E M} / 2$. Besides wire length, heating is also influenced by the wire geometry, placement in the human body, insulation, etc. The highest heating happens at locations where the electric field changes are the fastest [26,27]. The heating can lead to burn injuries [25,29].

As the varying magnetic field induces current in closed loops or components, electromagnetic energy is transformed into heat. According to Eqs. $(14,15)$,

$J=\int \sigma U_{i}^{2} d t$

The elevation of the object's temperature is

$\triangle T=\frac{1}{m C}\left(J-J_{t}\right)$

where $m$ is the mass, $C$ is the heat capacity, and $J_{t}$ is the heat dissipated from the object to the environment.

Although magnetic flux change due to gradient field switching is much less than that due to RF pulses, significant heating up solely due to gradient field switching was observed when a copper frame was placed $20 \mathrm{~cm}$ offcenter [28]. RF-induced heat due to eddy currents is difficult to predict accurately, but was shown to be minor by in vitro experiments [30,31].

\section{Disturbance to sensors and electronics}

Electromagnetic, inductive and capacitive sensors are not suggested for use in the MRI environment, or at least not in the close proximity to the imaging region, due to the mutual electromagnetic interferences. Other sensors and electronics may suffer from distortion to the characteristic curve or elevated noise due to the Hall effect, in addition to possible magnetic force, torque, induced current and heating.

Electronics inside the sensor and signals in transmission lines may be disturbed according to Eqs. $(5,6,16,17)$. We take a ratio to measure the disturbance by the scanner field to the detected signals:

$\beta_{S} \triangleq\left|\frac{\vec{I}_{i}}{I_{S}}\right| \quad$ or $\quad \beta_{S} \triangleq\left|\frac{\vec{U}_{i}}{U_{S}}\right|$

where $I_{i} / U_{i}$ is the induced signal on the sensor signal line, and $I_{S} / U_{S}$ is the amplitude of the signal by the sensor. In general, both $I_{i} / U_{i}$ and $I_{S} / U_{S}$ are time varying. Conservatively, $I_{i} / U_{i}$ takes the peak value of the induced signal. $I_{S} / U_{S}$ has to be defined based on the sensor's working range. By definition, $\beta_{S}$ is a noise-to-signal ratio.

\section{Disturbance to active components}

When active components based on electromagnetic principle are placed inside the MRI environment, their functioning is disturbed by the scanner magnetic field in addition to possible magnetic force, torque, induced current and heating effects.

To produce force or torque, electromagnetic motors rely on current passing through a magnetic field, which can be produced by a permanent magnet or a current carrying coil. When a motor is introduced into an MRI environment, the scanner field $\vec{B}(\vec{r})$ adds to the local magnetic field of the motor and thus changes the behavior of the motor. Hydraulic or pneumatic valves use combinations of coils, permanent magnets or springs to control the flow. Thus, they are also subject to disturbance by the magnetic field.

We take a ratio to demonstrate the disturbance by the scanner field to active components:

$\beta_{B} \triangleq\left|\frac{\vec{B}(\vec{r})}{B_{A}}\right|$

where $B_{A}$ is the amplitude of the equivalent magnetic field generated by coils or magnets in the active components. The ratio is achieved when $\vec{B}(\vec{r})$ and $\vec{B}_{A}$ are aligned. If the equivalent field of the actuator is time varying, $B_{A}$ should be defined according to the specific application, such as the root mean square value.

Active components are normally placed distant from the scanner, and, thus, the influence from the gradient and radio frequency fields can be neglected. 


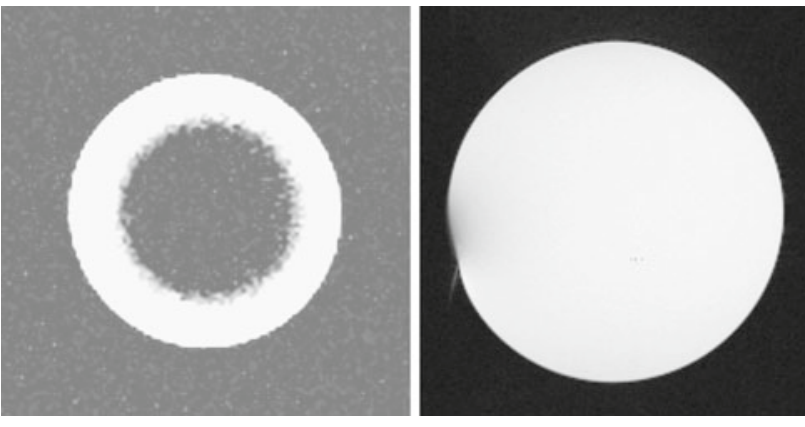

Fig. 5 Severe (left) and mild (right) image distortion due to material magnetization

\section{Deterioration of proper device functioning}

As a consequence of previous disturbances from the scanner field $\vec{B}(t, \vec{r})$, the desired function of the device can be deteriorated. We define a measure

$\beta_{f} \triangleq\left|\frac{p_{M R I}-p_{l a b}}{p_{l a b}}\right|$

where $p_{M R I}$ and $p_{l a b}$ are the performances of the device's desired function in the MRI environment and the laboratory, obtained with the same configuration. For example, if the device is position controlled and aims to guide the subject's arm to track a given position curve, then $p_{M R I}$ and $p_{l a b}$ are the actual curves obtained in the MRI environment and the laboratory, respectively.

\section{Localization error due to $B_{0}$ inhomogeneities}

When there is a distortion field $\Delta \vec{B}$ in the imaging area and $\triangle B_{z} \neq 0$, the localization information based on frequency and phase of the detected signals, as shown in Eq. (2), is shifted, resulting in localization error. The two other components of $\triangle \vec{B}, \triangle B_{x}$ and $\triangle B_{y}$, can affect phase contrast, just as the concomitant gradient terms do [19].

When $\Delta \vec{B}$ is aligned with $\vec{B}_{0}$ in the imaging area, the localization error $\Delta \vec{r}$ is maximal and satisfies

$\vec{G} \cdot \Delta \vec{r}=\triangle B$

When $\Delta \vec{B}$ increases, the localization error may go beyond the imaging volume and the signal will be lost (Fig. 5, left).

Given the voxel size $x_{v} \times y_{v} \times z_{v}$, suppose it is required that the maximal localization error is less than half of the voxel dimension in any direction. Therefore,

$|\triangle B|<\min \left\{G_{x} \frac{x_{v}}{2}, G_{y} \frac{y_{v}}{2}, G_{z} \frac{z_{v}}{2}\right\}$

The distortion field $\triangle \vec{B}(t, \vec{r})$ due to magnetized components and electric signals in active components and sensors can result in localization error as by Eq. (24).
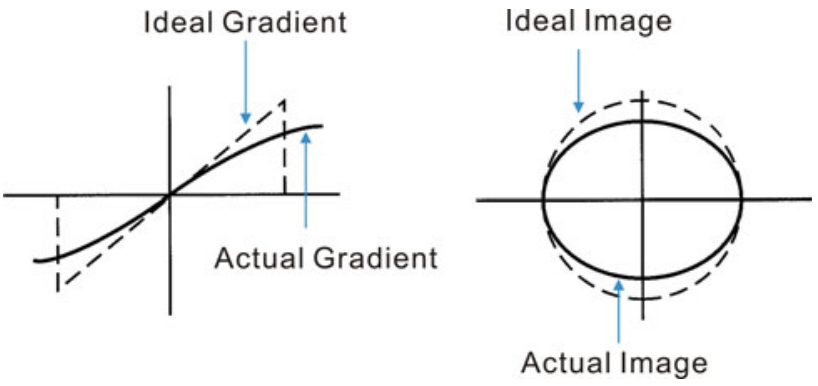

Fig. 6 Image distortion due to gradient distortion

Spatial encoding error due to gradient distortion

Gradient field distortion may result from the spatial gradients of the field generated by the induced current discussed in section c). Material magnetization solely due to the gradient field is negligible compared with that of the static field. A small gradient distortion may induce significant spatial encoding error since the distortion accumulates $\int \Delta \vec{G} \cdot d \vec{r}$ and produces the worst artifact at the boundary of the imaging volume, which is $\int \Delta \vec{G} \cdot d \vec{r}$ divided by $\vec{G}$ component-wise. (Fig. 6)

Although it is possible to pre-calibrate and correct the gradient non-linearity error [32], it is still preferred that no distortion field should be generated by the mechatronic system. If the gradient distortion varies temporally, the pre-calibration may not help.

A conservative upper bound for gradient distortion can be defined as

$|\triangle \vec{G}|<\min \left\{\frac{G_{x} \cdot x_{v}}{2 \cdot x_{\max }}, \frac{G_{y} \cdot x_{y}}{2 \cdot y_{\max }}, \frac{G_{z} \cdot x_{z}}{2 \cdot z_{\max }}\right\}$

Here, $\left(x_{\max }, z_{\max }, z_{\max }\right)$ is the extreme location of the imaging volume relative to the isocenter. This bound ensures that the worst spatial encoding error is smaller than half a voxel.

\section{Dark region due to signal dephasing}

The distortion field $\Delta \vec{B}$ may vary over the imaging volume. The spatial variation of $\triangle \vec{B}$ leads to signal dephasing [33,34]

$\beta_{\phi}=\gamma \cdot T_{E} \cdot \nabla|\triangle \vec{B}| \cdot \vec{r}_{v}$

where $\gamma$ is the gyromagnetic ratio, $T_{E}$ is the echo time, and $\vec{r}_{v}$ is the voxel size. This can cause signal void and appear as dark regions in the MR images.

\section{Image shading due to RF inhomogeneity}

Conductive materials or conductive loops in the imaging region can absorb the energy of RF pulses, leading to RF inhomogeneity. The neighboring tissues can therefore not reach a proper flip angle, and the signals will be reduced. 

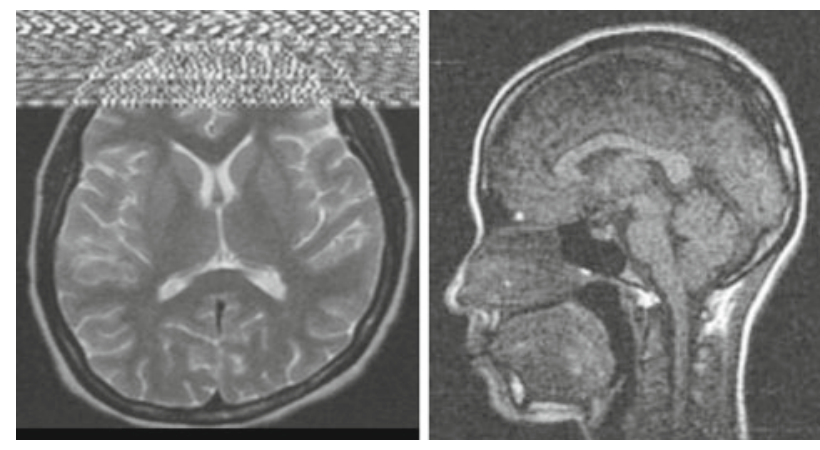

Fig. 7 Lines and speckles in images due to RF interferences

The artifacts are similar to those due to material magnetizations $[33,35]$. This artifact is significant and difficult to model. Therefore, conductive materials should not be used within the imaging region.

\section{Lines, speckles and noise due to RF interferences}

If the distortion field $\triangle \vec{B}(t)$ contains frequency components in the bandwidth of the RF pulses, it results in lines and speckles on the obtained images (Fig. 7) [36]. Hence, electromagnetic interference in the RF bandwidth must be prevented.

The radio frequency distortion field mainly results from magnetically induced currents (Fig. 4), sensor and actuator electronics, or noise brought into the MRI environment by wires due to the antenna effect.

\section{False fMRI signal}

Dynamic distortion fields outside the RF bandwidth may lead to MRI signal changes, which can be mis-attributed to changes in neural activation.

This interference mainly results from driving currents in electrically active components and noise brought into the MRI environment by the antenna effect. An important point is that electromagnetic interference is often correlated with the task. For example, an encoder moving close to the imaging area during the investigated task may disturb the fMRI signals.

\section{Design principles for MRI-compatibility}

Formulation of MRI-compatibility

The MRI-compatibility criteria are transformed into Table 2 based on the analysis on mutual interferences between mechatronic devices and the MRI system.
The qualitative description of MRI-compatibility is not measurable. In contrast, each interference is evaluated quantitatively by a numerical measure in Table 2 . Although eddy current in (c) can not be explicitly calculated, the consequences, heating (d) and image deterioration (i), (k), (l), indirectly measure how much influence eddy currents have on the mechatronic device.

The MRI-compatibility thresholds will vary depending on the application. For example, a larger voxel size tolerates larger localization errors, smaller $T_{E}$ brings less dephasing artifacts, and fMRI procedures are subject to a wide range of noise which may not be so problematic for anatomical MRI scans. Besides, the final mechatronic system is a balance of cost, desired function and MRI-compatibility. Therefore, the user needs to compromise competitive requirements and define specific acceptance thresholds for specific applications. In Table 2, each acceptance threshold can be adjusted to fit specific applications.

\section{Design principles}

The design procedure can be broken down into several parts. For each part, the MRI-compatibility criteria in Table 2 help to prevent potential problems with safety, device functioning or MRI image quality. While the MRI-compatibility criteria are based on interactions of the MRI system with mechatronic devices, a component-wise categorization as in Table 3 is more convenient for device development.

\section{The MRI system}

MRI-compatibility is linked to a specific MRI environment and special condition, such as the imaging parameters and the placement of the mechatronic component within this environment. Therefore, the following parameters of the MRI system should be known (Table 1) prior to the design process:

- Static magnetic field $\vec{B}_{0}$ : nominal amplitude and homogeneity in the imaging area, field distribution in the MRI scanner room, maximum gradient $|\vec{\nabla}| \vec{B}_{0}||$, maximum $\left|\vec{B}_{0}\right| \cdot|\vec{\nabla}| \vec{B}_{0}||$

- Gradient system $\vec{G}$ : amplitude, slew rate, rise time, switching frequency

- Pulsed radio frequency field: amplitude, nominal frequency, bandwidth, for both the transmitting $\vec{B}_{1}+$ and the receiving $\vec{B}_{1}-$

- Scanner bore dimension

- Intended location of equipment under development

- The type of penetration panel available (filters, waveguides, surrounding equipment, etc.). 
Table 2 MRI-compatibility criteria based on the mutual interferences between the MRI system and mechatronic devices

\begin{tabular}{|c|c|c|}
\hline Interference & Components Involved & MRI-Compatibility Criteria \\
\hline (a) Magnetic force & $\begin{array}{l}\text { Magnetized components } \\
\text { Current lines }\end{array}$ & $\beta_{F}<\beta_{F_{-} t h}$ \\
\hline (b) Magnetic torque & $\begin{array}{l}\text { Magnetized components } \\
\text { Current loops }\end{array}$ & $\beta_{\tau}<\beta_{\tau_{-} t h}$ \\
\hline (c) Induced current & $\begin{array}{l}\text { Conductive components } \\
\text { Conductive loops }\end{array}$ & $I_{i}<I_{i_{-} t h}^{\dagger}$ \\
\hline (d) Heating & $\begin{array}{l}\text { Conductive components } \\
\text { Conductive loops }\end{array}$ & $J<J_{t h}^{\dagger}$ \\
\hline & Open wire & No open wire or $L \ll \lambda_{E M} / 2$ \\
\hline (e) Disturbance to sensors and electronics & $\begin{array}{l}\text { Sensors } \\
\text { Electronics }\end{array}$ & (a) (b) (c) (d) \& $\beta_{S}<\beta_{S_{-} t h}$ \\
\hline (f) Disturbance to active components & Active components & (a) (b) (c) (d) \& $\beta_{B}<\beta_{B_{-} t h}$ \\
\hline (g) Device function deterioration & The whole system & (a) (b) (c) (d) (e) (f) $\& \beta_{f}<\beta_{f_{-} t h}$ \\
\hline (h) Localization error due to $\Delta \vec{B}$ & $\begin{array}{l}\text { Magnetized components } \\
\text { Actuator signals } \\
\text { Sensor signals }\end{array}$ & $|\triangle B|<\triangle B_{t h}$ \\
\hline (i) Spatial encoding error due to $\Delta \vec{G}$ & $\begin{array}{l}\text { Conductive components } \\
\text { Conductive loops }\end{array}$ & $|\triangle G|<\Delta G_{t h}$ \\
\hline (j) Dark region due to signal dephasing & $\begin{array}{l}\text { Magnetized components } \\
\text { Actuator signals } \\
\text { Sensor signals }\end{array}$ & $\beta_{\phi}<\beta_{\phi_{-} t h}$ \\
\hline (k) Image shading due to RF inhomogeneities & $\begin{array}{l}\text { Conductive components } \\
\text { Conductive loops }\end{array}$ & Avoid components in imaging region $*$ \\
\hline (1) Lines, dots, noise due to RF interferences & $\begin{array}{l}\text { Conductive components } \\
\text { Conductive loops } \\
\text { Actuator signals } \\
\text { Sensor signals } \\
\text { Transmission wires }\end{array}$ & $\beta_{\omega}<\beta_{\omega_{-} t h}$ shielding \\
\hline (m) False fMRI interpretation & $\begin{array}{l}\text { Actuator signals } \\
\text { Sensor signals } \\
\text { Transmission wires }\end{array}$ & $\left|\triangle B_{t}\right|<\Delta B_{t_{-} t h}$ shielding \\
\hline
\end{tabular}

$\dagger$ There is no general analytic solution ${ }^{\star}$ Some special applications may allow a certain extent of artefacts

Table 3 The MRI-compatibility matrix

* Aspect ( $\mathrm{g}$ ) of Table 2 is not included, since it is the overall consequence of (a)-(f)

\begin{tabular}{lllll}
\hline & $\begin{array}{l}\text { Static field } \vec{B}_{0} \text { gradient } \\
\vec{\nabla}\left|\vec{B}_{0}\right|\end{array}$ & $\begin{array}{l}\text { Gradient field } \\
\vec{G}, \frac{\partial \vec{G}(t) \cdot \vec{r}}{\partial t}\end{array}$ & $\begin{array}{l}\text { RF field } \\
\vec{B}_{1}, \frac{\partial \vec{B}_{1}(t)}{\partial t} \vec{E}(t)\end{array}$ & fMRI \\
\hline$\chi$ : Magnetic susceptibility & (a) (b) (h) (j) & - & - & \\
$\sigma:$ Electrical conductivity & - & (c) (d) (i) & (c) (d) (k) (l) & \\
Current line & (a) & - & - & \\
Transmission wire & (a) & - & (l) & (m) \\
Current loop & (b) & - & - & \\
Conductive loop & (b) & (c) (d) (i) & (c) (d) (k) (l) & \\
Open wire & - & - & (d) & \\
Electronics & - & (e) & (e) (i) (l) & (m) \\
Sensor & (a) (b) (h) (j) & (c) (d) (e) (i) & (c) (d) (e) & (m) \\
Active components & (a) (b) (f) (h) (j) & (c) (d) (i) & (c) (d) & (m) \\
\hline
\end{tabular}


Although the scanner bore dimension is not mentioned in the MRI-compatibility matrix, it is a fundamental limitation for mechatronic devices, as well as the experimental paradigms to be investigated.

\section{Structure and materials}

Various plastic materials are magnetically and electrically inert and are thus preferred as construction materials. However, their mechanical properties are often poor and metal has to be used in some applications.

To avoid resonance heating, there must be no open conductive wire. When materials with high magnetic susceptibility are brought into the MRI environment, the magnetic force/torque brings potential safety hazards. Hence, these materials should be generally avoided and only used in the MRI environment after test.

Since analytic solutions to Eqs. $(17,19)$ just exist in a few special cases, it is difficult to guide the design process by quantitative thresholds on eddy currents and the related heating and induced magnetic field. Therefore, generally we should try to limit use of conductive materials and prevent these phenomena. Conductive components or closed loops must not be placed in the imaging area since they can induce eddy currents and lead to significant local artifacts [23]. Outside the scanner bore, conductive materials with low magnetic susceptibility can be used for static components. They might be used for closed loop structures or moving parts if it has been demonstrated no violation to the MRI-compatibility.

Schenck [5] performed a detailed study on physical properties of selected materials and their roles in MRI applications. The study has heavily benefited subsequent work and remains a fundamental reference. The range of susceptibility values acceptable for mechatronic devices depends on specific applications, particularly on magnetic force, torque and magnetization. It should be mentioned that machining of a material may change its magnetic properties.

Suppose a component is placed at $\vec{r}$ relative to the head coil, and the magnetic saturation point is not reached. According to Eqs. (10, 13), we have

$$
\left\{\begin{array} { l } 
{ \beta _ { F } < \beta _ { F _ { - } t h } } \\
{ \beta _ { \tau } < \beta _ { \tau _ { - } t h } } \\
{ | \triangle B | < \Delta B _ { t h } }
\end{array} \Rightarrow \left\{\begin{array}{l}
\frac{|\chi|}{\rho}<\frac{\mu_{0} g}{\max \left|\vec{B}_{0}\right| \cdot|\vec{\nabla}| \vec{B}_{0}||} \beta_{F_{-} t h} \\
\frac{|\chi|}{\rho}<\frac{\mu_{0} g l}{\max |\vec{B}|^{2}} \beta_{\tau_{-} t h} \\
|\chi|<\frac{2 \pi|\vec{r}|^{3}}{|\vec{B}(\vec{r})| V} \Delta B_{t h}
\end{array}\right.\right.
$$

where $l$ is the component's largest dimension, and $V$ the volume. Equation (28) limits possible selection of materials to be used for this component, under the MRI-compatibility requirements.
In special applications where a certain extent of artifact is acceptable, experiments should be done carefully on the specific MRI systems.

\section{Active components and sensors}

Various actuation principles have been evaluated in the MRI environment, such as pneumatic [37-39], hydraulic [40,41], electromagnetic [20], cable-driven [42], piezo-electric [43, 44] and electro-rheological [45]. Each actuation technique has its special advantages $[3,6]$.

The location of an active component inside the scanner room has to be carefully considered to make sure that its interactions with the MRI system are weak enough. In order not to be affected by the scanner field, an active component should be kept at a minimal distance away from the volume of interest:

$|\vec{r}|>\max \left|\vec{r}_{t h}\right|, \quad$ where $\left|\vec{B}_{0}\left(\vec{r}_{t h}\right)\right|=\beta_{B} B_{A}$

On the other hand, given the component's magnetic moment $\vec{M}$, its distance to the imaging area has to fulfill

$|\triangle B|<\Delta B_{t h} \Rightarrow|\vec{r}|>\left(\frac{\mu_{0}|\vec{M}|}{2 \pi} \frac{1}{\triangle B_{t h}}\right)^{\frac{1}{3}}$

The threshold will be the higher of the two values.

If $\vec{M}$ is time varying, it will produce noise in MRI images. Shielding is necessary in this case.

Optical sensors are popular [3,7,20], but they have to be applied in a proper way to avoid high-frequency disturbances to the MRI system [7]. A load cell was used in [46] to measure force. The optical measurement principle is MRI-compatible itself, but the compatibility of components of the sensor has to be verified.

\section{Electronics and signal transmission lines}

Transmission lines send currents to drive active components inside the MRI environment and send out detected sensor signals. The transmission lines should be arranged to avoid any loops. The human subject should never be put as part of any conductive loops.

To avoid scanner interferences to the carrying signals and also to avoid the antenna effect that may carry noise from the control room into the scanner room, transmission lines must be well shielded, and the shielding layer should be grounded to the shielding of the scanner room. If electronics stay inside the MRI room, it is suggested that they are put inside a Faraday cage to prevent mutual interference with the scanner. 


\section{Guidelines for electromagnetic compatibility}

Electromagnetic interference is often subtle, and the source of interference may not be so straightforward to determine. Following guidelines can help to eliminate or prevent electromagnetic interference [47]

- Place interfering or sensitive components into a Faraday cage for shielding against high-frequency noise

- Select materials with high magnetic susceptibility to shield against low-frequency magnetic fields, but be sure to secure them distant enough from the scanner

- Minimize the number of interfaces, where signals and power lines enter the shielding

- Filter those interfaces to low impedance ground

- Reduce common mode noise in differential signals by twisted pair cables

- Utilize ferrite cores and common mode chokes to further suppress common mode noise

- Employ shielded cables and connectors with a low impedance connection

- Implement radio frequency filters on power and data signals at the level of the penetration panel, which provides a stable low impedance earthed-ground

- Avoid ground loops

- Separate power electronics and logic electronics

- Include a ground plane on printed circuit boards

\section{Evaluation of MRI-compatibility}

In this section, we propose procedures to evaluate whether a device is MRI-compatible, with respect to safety, functioning of the device and MRI image quality.

Safety and device functioning

\section{Force measurement}

The magnetic force can be measured relatively to the component's gravitational force [10]. When $\beta_{F}<\beta_{F_{-} t h}=1$, it is assumed in [10] that the risk by the magnetic force of a component is less than the risk by its gravity.

\section{Torque measurement}

A magnetic torque measurement device can be found in [11]. When $\beta_{\tau}<\beta_{\tau_{-} t h}=1$, i.e., the maximal value of $\vec{\tau}_{M}$ is less than the gravity torque $\tau_{g}$, it is assumed in [11] that the magnetically induced deflection torque is less than the worst case torque on the device due to gravity.

\section{Heating measurement}

In the design principles section, we prohibit conductive materials and closed loops in the imaging area. Consequently, heating measurements are not necessary. For very special applications in which the heating measurement has to be performed, a standard method to measure radio frequency induced heating in MRI is defined in ASTM F2182-02a, 2002, and the study in [28] gives the procedures to switch off the RF pulses and measure the heating effect due to the switching gradient field.

\section{Disturbance to sensors and electronics}

The electromagnetic environment in the MRI room may affect sensors and electronics in multiple ways, such as distortion of the voltage-current characteristic curve and elevated noise. Therefore, it is recommended that the components should be calibrated at their desired working position with the correct orientation in the MRI environment, to make sure they work in a proper way. To evaluate the noise, we can record the detected signals and take the standard deviation as the noise $I_{i}$ in Eq. (21). $I_{S}$ takes the sensor signal range. A typical threshold is $5 \%$.

\section{Disturbance to active components and device function}

After the safety aspects, i.e., force, torque and heating, have been guaranteed, the device is allowed to enter the MRI environment for further evaluation. Disturbance to active components is closely related to deterioration of device function, and thus, it is reasonable to test Eq. (23) instead of Eq. (22). Nevertheless, active components can still be solely tested according to their specifications. We again take $5 \%$ as an acceptable threshold for both Eqs. (23) and (22).

\section{MRI image quality}

Image quality is usually assessed using a standard phantom $[40,41,44,45]$. After each scan, the MRI system processes the data and outputs MRI images, and we can check whether the image quality has been affected.

We propose that the full MRI experiment can be carried out in the following six steps:

1. Phantom only

The scan is performed when the device is not inside the scanner room. MRI images from this step serve as the baseline of the entire experiment. At least ten minutes of data should be collected, so that temporal drift can be estimated.

2. Device disconnected

The device is placed at its desired working location in 
the scanner room but has no connection going out of the scanner room. MRI images from this step tell whether magnetic susceptibility introduces localization error or eddy current induces noise into the imaging procedure.

3. Device connected

The device is placed at its desired working location in the scanner room, with all the transmission lines connected to the parts out of the scanner room, but not powered. If there is no image artifact from the previous step, images from this step will reveal whether the transmission lines carry noise from the control room into the scanner room.

4. Device powered

The device is placed at its desired working location in the scanner room, with all the transmission lines connected to the parts out of the scanner room and powered, but not performing any task. If there is no image artifact from previous steps, images from this step will reveal whether the electric signals have caused image artifacts.

5. Device functioning

The device is placed at its desired working location in the scanner room, with all the transmission lines connected to the parts out of the scanner room, powered, and performing the desired task. If there is no image artifact from previous steps, images from this step tell whether functioning of the device causes image problems, such as false fMRI signal and eddy currents induced inside moving conductive parts.

The evaluation of device functioning as described in section 2.3.g) can be integrated into this step.

6. Phantom only again (device removed)

The scan is taken after the device is completely removed from the scanner room. Comparison of images from this step and the first step indicates the temporal stability of the scanner system and serves as a criterion for other comparisons.

Electromagnetic interference may lead to different image artifacts with different MRI or fMRI sequences, such as localization errors in phase encoding direction for Cartesian EPI (Echo-Planar Imaging), blurring in spiral EPI and localization errors in readout direction in GRE (Gradient-Recalled Echo). Therefore, MRI-compatibility with one scan sequence on a certain scanner system cannot guarantee a device being MRI-compatible again with a different scan sequence or on a different scanner system. Consequently, all the experimental steps must be performed on the same MRI system with exactly the same scan sequence and parameters as the actual study in which the device is to be used. Auto pre-scan functions should be disabled, so that possible image artifacts can be directly traced back to electromagnetic interferences. Enabled auto pre-scan functions can react to electromagnetic interferences and confound the image artifacts.

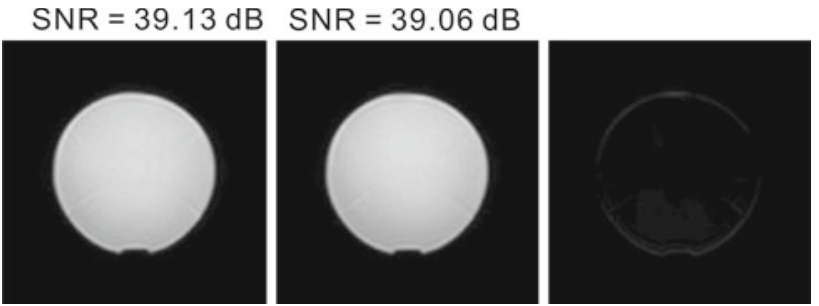

Fig. 8 An example for SNR and subtraction of MRI images. Left phantom only; middle device functioning; right subtraction of the previous images. The SNR changed by $0.18 \%$

Widely used MRI image evaluation methods in literature are image subtraction and signal-to-noise-ratio (SNR) comparison, which visually and quantitatively show differences between images acquired before and after the mechatronic device is introduced into the MRI environment (Fig. 8). The SNR value of one image is typically calculated as [33]

$\mathrm{SNR}=\frac{0.66 \times \text { mean signal }}{\text { mean of noise region standard deviations }}$

The scalar 0.66 is the Rayleigh distribution correction factor. It has to be noted that this method is not suitable for parallel MRI images, such as fMRI images obtained with the SENSE $^{1}$ technique [48]. Another method acquires two identical images consecutively, subtracts them and then calculates the SNR by [33]

$\mathrm{SNR}=\frac{\sqrt{2} \times \text { mean signal in image ROI }}{\text { standard deviation in subtraction image ROI }}$

The ROI (region of interest) for signal and noise must be the same. In both methods, it is recommended that the ROI should cover $75 \%$ of the phantom area. Additionally, it is common to present $\mathrm{SNR}$ values in $\mathrm{dB} .^{2}$

Another quantitative measure was proposed in [49]. An image deterioration factor is calculated as

$\epsilon_{z}^{T_{i}}=\frac{100}{2^{2 p_{d}}} \cdot \frac{1}{r_{x} \cdot r_{y}} \cdot \sum_{\substack{x=1, \ldots, r_{x} \\ y=1, \ldots, r_{y}}}\left(p_{x y}^{T_{1}}-p_{x y}^{T_{i}}\right)^{2}$

Here, $z$ is the slice number, $i$ is the test step number, $p_{d}$ is the pixel depth, $r_{x}$ and $r_{y}$ are the image resolutions along the $\mathrm{x}$ and $\mathrm{y}$ axes, and $p_{x y}^{T_{i}}$ ranging from 0 to $2^{2 p_{d}}-1$ is the pixel value with index $(x, y)$ in the image obtained at test step $T_{i}$. Averaging $\epsilon_{z}^{T_{i}}$ over all slices gives a global image deterioration factor.

Furthermore, we propose a third comparison method. In fMRI applications, each scanning session takes multiple images of the same slice. For one voxel in this slice (Fig. 9), its temporal-signal-to-noise-ratio (tSNR) is the ratio of the

\footnotetext{
${ }_{1}^{1}$ Sensitivity encoding for fast MRI.

2 Decibel: (number in $\mathrm{dB})=20 \log _{10}$ (number in decimal).
} 


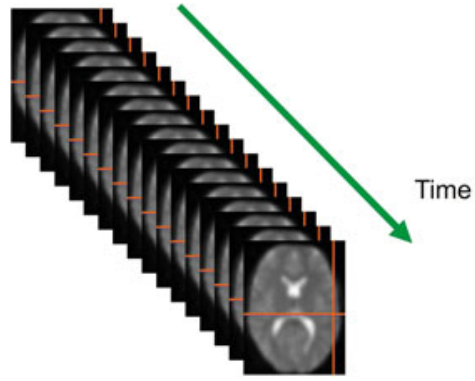

Slice time series

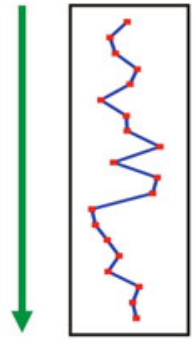

voxel time series

Fig. 9 Left time series of one slice; right time series of a voxel

standard deviation of its time series over the mean of its time series. The tSNR of this slice is the mean of tSNRs over a selected region, as defined in Eq. (33).

$\mathrm{tSNR}=$ mean $\frac{\text { standard deviation of voxel time series }}{\text { mean of voxel time series }}$

To check whether the SNR and tSNR values have been "significantly" changed, statistical tests are employed. We suggest t-test, z-test and the Kolmogorov-Smirnov test [50-52].

In summary, image subtraction detects localization errors, spatial encoding errors and signal voids due to dephasing, the SNR comparison detects background noise, and the tSNR detects low-frequency noise and false fMRI signals.

Standard MRI phantom images have been used to evaluate the influence from mechatronic devices on the MRI system. They can be interpreted in a straightforward manner by people working in this interdisciplinary field with different background, such as engineers, MRI experts and medical doctors. There are more specialized tests available, such as acquisition and analysis of $B_{0}$ and $B_{1}$ field maps [53-56]. In case that suspicious spatial distortion is observed in visual inspection or image subtraction in the proposed test procedures, gradient linearity tests using special phantoms are suggested in [57].

\section{Design and evaluation of an MRI-compatible manipulandum}

Following the design principles in the previous section, we design a one degree-of-freedom (DOF) manipulandum to perform interactive reaching movement with the arm of a subject lying supine on the scanner bed during fMRI procedures. The fMRI sequence is a $T_{2}$-weighted, single-shot, field echo, echo-planar imaging (EPI) sequence (TR: $3000 \mathrm{~ms}$, echo time TE: $40 \mathrm{~ms}$, SENSE factor: 2 ). The desired voxel size is $1 \mathrm{~mm} \times 1 \mathrm{~mm} \times 3 \mathrm{~mm}$, and $\triangle B=20 \mu \mathrm{T}$ by Eq. (25).

The system concept is shown in Fig. 10. A hydraulic cylinder actuates the interaction with the human arm. Force and

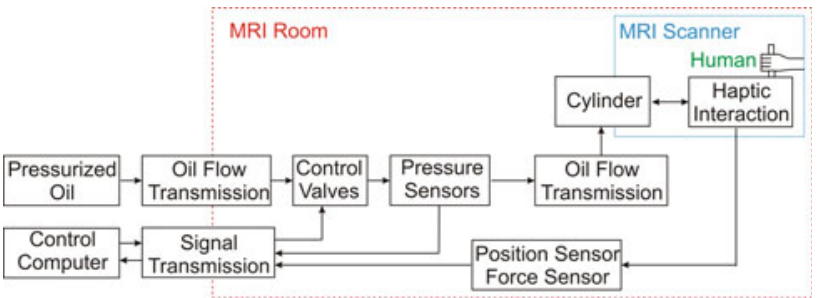

Fig. 10 Diagram of the MRI-compatible manipulandum

position of the hand are detected by sensors and sent out of the MRI room to the control computer. The computer controls the valve to regulate the hydraulic power generated by the pump placed outside the MRI room and transmitted into the MRI room via plastic hoses and drives the actuation cylinder.

Construction materials are mainly plastics. The force sensor adopts an optical principle [20], which is MRI-compatible in nature. Violation of MRI-compatibility may result from the control valve, the hydraulic cylinder, the position sensor and electric signal transmission lines. The control valve unit is electromagnetically active and stays at the corner of the MRI room, far away from the scanner bore. The hydraulic cylinder is placed close to the scanner bore and should be made of metal metal with low magnetic susceptibility to sustain high pressure. The position sensor is also placed close to the scanner, and connects to the control computer outside the scanner room.

\section{Cylinder material}

The cylinder is close to the imaging region, especially the moving piston. Therefore, the material must have very low magnetic susceptibility. The volume of the cylinder housing is $V_{1}=7.0 \times 10^{-4} \mathrm{~m}^{3}$, and the volume of the cylinder piston is $V_{2}=3.7 \times 10^{-5} \mathrm{~m}^{3}$. According to Eq. (28), we obtain

$$
\left\{\begin{array}{l}
\frac{|\chi|}{\rho}<2.6 \times 10^{-7} \beta_{F_{-} t h} \\
\frac{|\chi|}{\rho}<4.5 \times 10^{-7} \beta_{\tau_{-} t h} \\
|\chi|<6.0 \times 10^{-5} \text { for } V_{1},|\chi|<1.1 \times 10^{-3} \text { for } V_{2}
\end{array}\right.
$$

To be conservative, we take the thresholds $\beta_{F_{-} t h}$ and $\beta_{\tau_{-} t h}$ to be $10 \%$ of the given values in the previous section, i.e., $\beta_{F_{-} t h}=\beta_{\tau_{-} t h}=0.1$. Five materials, i.e., aluminum $\left(\chi=2.07 \times 10^{-5}, \rho=2.7 \times 10^{3} \mathrm{~kg} / \mathrm{m}^{3}\right)$, copper $\left(\chi=-9.63 \times 10^{-6}, \rho=8.92 \times 10^{3} \mathrm{~kg} / \mathrm{m}^{3}\right)$, brass $\left(\chi=-8.63 \times 10^{-6}, \rho=8.92 \times 10^{3} \mathrm{~kg} / \mathrm{m}^{3}\right)$, zinc $\left(\chi=-1.57 \times 10^{-5}, \rho=7.13 \times 10^{3} \mathrm{~kg} / \mathrm{m}^{3}\right)$ and bronze $\left(\chi=-8.79 \times 10^{-7} \rho=8.79 \times 10^{3} \mathrm{~kg} / \mathrm{m}^{3}\right)$ fulfill the above limitations [5].

In the worst case, the direction of the piston movement is parallel to $\vec{B}_{0}$ and perpendicular to $\vec{B}_{1}$ (Fig. 11). Hence, eddy currents are mainly induced by magnetic flux changes due to the spatial gradient of $\vec{B}_{0}$. For a fixed dimension of 


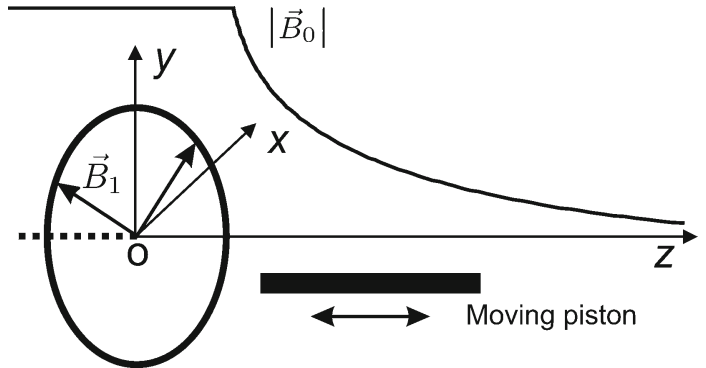

Fig. 11 The piston moves in parallel with the $\vec{B}_{0}$ and perpendicular to $\vec{B}_{1}$

the piston, the induced potential is constant. Eddy currents are minimal when the conductance of the piston is minimal. Therefore, we use bronze for the piston.

The piston has a simple geometry. The induced eddy current and magnetic field can be calculated [58]. Since the bronze piston moves slowly, less than $0.3 \mathrm{~m} / \mathrm{s}$, the distorting magnetic field generated by the eddy currents is far smaller than $\triangle B$.

Location of the control valve unit

The control valve unit is active, and it contains ferromagnetic parts. Therefore, it has to stay away from the scanner, and we need to determine the minimal distance.

We again take $\beta_{F}$ th $=0.1$. The torque is not important here and we can ignore it. Inserting the magnetic susceptibility and density values of stainless steel into Eq. (28), we obtain

$$
\left\{\begin{array} { l } 
{ \beta _ { F } < \beta _ { F _ { - } t h } } \\
{ | \triangle B | < \triangle B _ { t h } }
\end{array} \Rightarrow \left\{\begin{array}{l}
|\vec{r}|>5.6 \\
|\vec{r}|>1.25
\end{array}\right.\right.
$$

Therefore, we take the hose length between the cylinder and the control valve to be $6 \mathrm{~m}$.

The model in Eq. (3) is utilized to calculate the magnetic field and its spatial gradient. The scanner field here is below $4.1 \mathrm{mT}$ according to the model and is below $0.3 \mathrm{mT}$ according to the field map provided by Philips [17]. The inconsistency results from the modeling error, but we are on the safe side with the model.

The solenoid pair inside the valve produces a magnetic field. The modeling and calculation of the solenoid field propagation is time-consuming and may be inaccurate since the valve unit's metal housing shields the field to a certain extent. Instead, we simply take a Hall sensor to measure the magnetic field surrounding the valve when it is working under full load. The amplitude is much lower than the threshold value of $20 \mu \mathrm{T}$ at $6-\mathrm{m}$ distance.

The supply voltage for the valve is $12 \mathrm{~V}$, and the maximal current is $2.3 \mathrm{~A}$. The power lines are more than $6 \mathrm{~m}$ away from the scanner. Simple calculations confirm no violation of MRI-compatibility.

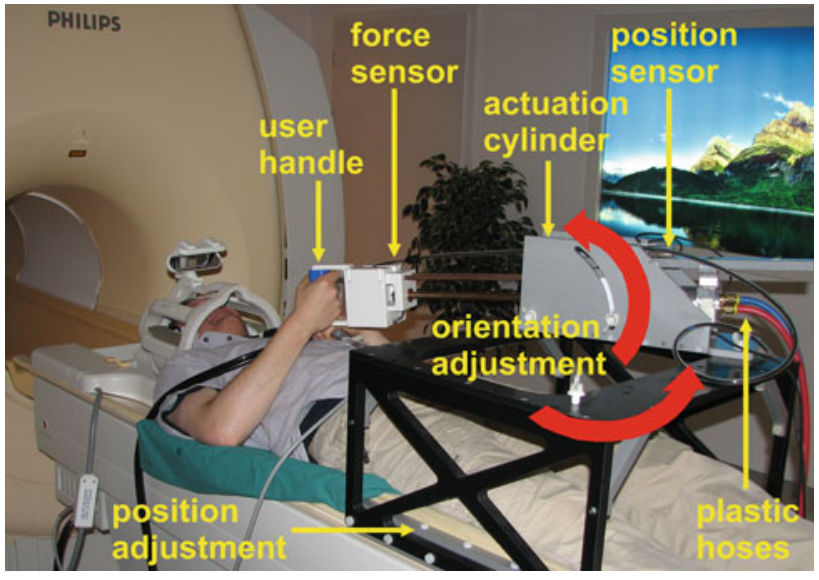

Fig. 12 The MRI-compatible manipulandum

\section{Position sensors}

To accurately measure the position, an optical encoder, LIDA 279 by Heidenhain, is used. Additionally, a potentiometer, MTP-L 22 by Resenso, is taken as a redundant position sensor for safety. The encoder scale tape is fixed, and the encoder reader moves slowly with the piston. The scale tape is made of MRI-compatible materials. With similar procedures as the previous section, the encoder reader is confirmed MRI-compatible.

In the literature, it has been reported that a potentiometer may create problems [50]. The conductive foil of our potentiometer is made of a special material, and no problem has been observed. The potentiometer may collect noise from the scanner system, but this is acceptable for a redundant sensor.

\section{Electromagnetic compatibility}

Last but not the least, the guidelines for electromagnetic compatibility are followed. They are important to guarantee good MRI image quality.

The realized device is shown in Fig. 12. It has been confirmed by MRI-compatibility tests that the one-DOF manipulandum is MRI-compatible in our Philips Achieva 3.0T scanner, located at the entry of the scanner bore, with the applied sequence [41].

\section{Conclusion}

We have studied possible mutual interferences between mechatronic systems with an MRI system and formulated numerical measures for each interference. A list of quantitative requirements were organized into an "MRI-compatibility matrix" for mechatronic devices to work compatibly with MRI procedures. Based on this matrix, design principles for 
the development of MRI-compatible mechatronic systems were proposed. These design principles ensure that potential safety, device functioning and image quality issues can be considered and dealt with already in the design phase. Test methods were proposed to evaluate whether the mechatronic device is indeed compatible with MRI procedures. Finally, the proposed design principles were successfully applied to the design of an MRI-compatible manipulandum. We hope that these guidelines will serve as a basis for the design and evaluation of mechatronic devices for various MRI/fMRI applications, and encourage designers to apply these tests and report these compatibility data in future publications.

Acknowledgments The authors would like to thank Christoph Hollnagel and Andreas Brunschweiler from the Sensory-Motor Systems Lab, ETH Zurich, Natalia Estévez and Prof. Dr. Spyros S. Kollias from the Institute of Neuroradiology, University Hospital Zurich, Dr. Roger Lüchinger and Prof. Peter Böiger from the MR center, University and ETH Zurich, for their help, support and inspiration to this work. Roger Gassert is supported by the NCCR Neural Plasticity and Repair of the Swiss National Science Foundation. This work was supported by the Swiss National Science Foundation NCCR on Neural Plasticity and Repair, project P8 Rehabilitation Technology Matrix, as well as ETH Research Grant TH-34 06-3 MR-robotics.

\section{References}

1. Gassert R, Burdet E, Chinzei K (2008) Opportunities and challenges in MR-compatible robotics. IEEE Eng Med Biol Mag 27(3): 15-22

2. Tsekos NV, Khanicheh A, Christoforou E, Mavroidis C (2007) Magnetic resonance-compatible robotic and mechatronics systems for image-guided interventions and rehabilitation: a review study. Annu Rev Biomed Eng 9:351-387

3. Yu N, Riener R (2006) Review on MR-compatible robotic systems. In: The IEEE/RAS-EMBS international conference on biomedical robotics and biomechatronics (BioRob), Pisa, Italy

4. Chinzei K, Kikinis R, Jolesz FA (1999) MR compatibility of mechatronic devices: design criteria. In: The international conference on medical image computing and computer-assisted intervention

5. Schenck JF (1996) The role of magnetic susceptibility in magnetic resonance imaging: MRI magnetic compatibility of the first and second kinds. Med Phys 23(6):815-850

6. Gassert R, Yamamoto A, Chapuis D, Dovat L, Bleuler H, Burdet E (2006) Actuation methods for applications in MR environments. Concepts Magn Reson B Magn Reson Eng 29(4):191-209

7. Gassert R, Chapuis D, Bleuler H, Burdet E (2008) Sensors for applications in magnetic resonance environments. IEEE/ASME Trans Mechatron 13(3):335-344

8. A primer on medical device interactions with magnetic resonance imaging systems. The US Food and Drug Administration (FDA) (1997)

9. MR safety and MR compatibility: test guidelines for signa $\mathrm{SP}^{\mathrm{TM}}$, Version 1.0. GE Medical Systems (1997)

10. Standard test method for measurement of magnetically induced displacement force on medical devices in the magnetic resonance environment. ASTM F2052-06e1, 2006

11. Standard test method for measurement of magnetically induced torque on passive implants in the magnetic resonance environment. ASTM F2213-06, 2006
12. Standard test method for evaluation of MR image artifacts from passive implants. ASTM F2119-07, 2007

13. Schaefers G (2008) Testing MR safety and compatibility. IEEE Eng Med Biol Mag 27(3):23-27

14. Guidance for industry and FDA staff-establishing safety and compatibility of passive implants in the magnetic resonance (MR) environment. The US Food and Drug Administration (FDA) (2008)

15. Standard test method for evaluation of MR image artifacts from passive implants. ASTM F 2119-01, 2001

16. Gassert R, Burdet E, Chinzei K (2008) MRI-compatible robotics. IEEE Eng Med Biol Mag 27(3):12-14

17. Technical description: Intera 1.5T Release 2.5 series, Achieva 1.5T / 3.0T / XR Release 2.5, Panorama HFO Release 2.5 series. Philips Medical Systems Nederland (2007)

18. Wilde JD, Price D, Williams J, Delakis I, Renaud C (2004) Comparative specications of four 3T MRI systems. Medicines and Healthcare products Regulatory Agency, Department of Health, UK, techniacl report

19. Bernstein MA, Zhou XJ, Polzin JA, King KF, Ganin A, Pelc NJ, Glover GH (1998) Concomitant gradient terms in phase contrast MR: analysis and correction. Magn Reson Med 39(2):300-318

20. Riener R, Villgrattner T, Kleiser R, Nef T, Kollias S (2005) fMRIcompatible electromagnetic haptic interface. In: The IEEE-EMBS annual international conference of the engineering in medicine and biology society (EMBS), vol 7, pp 7024-7027

21. Chapuis D (2009) Application of ultrasonic motors to MR-compatible haptic interfaces. $\mathrm{PhD}$ Thesis

22. Graf H, Lauer UA, Schick F (2006) Eddy-current induction in extended metallic parts as a source of considerable torsional moment. J Magn Reson Imaging 23(4):585-590

23. Camacho CR, Plewes DB, Henkelman RM (1995) Nonsusceptibility artifacts due to metallic objects in MR imaging. J Magn Reson Imaging 5(1):75-88

24. Standard test method for measurement of radio frequency induced heating near passive implants during magnetic resonance imaging. ASTM F2182-02a, 2002

25. Konings MK, Bartels LW, Smits HF, Bakker CJ (2000) Heating around intravascular guidewires by resonating RF waves. J Magn Reson Imaging 12(1):79-85

26. Duru F, Luechinger R, Scheidegger MB, Luscher TF, Boesiger P, Candinas R (2001) Pacing in magnetic resonance imaging environment: clinical and technical considerations on compatibility. Eur Heart J 22(2):113-124

27. Lchinger RC (2002) Safety aspects of cardiac pacemakers in magnetic resonance imaging. $\mathrm{PhD}$ thesis

28. Graf H, Steidle G, Schick F (2007) Heating of metallic implants and instruments induced by gradient switching in a 1.5-Tesla whole-body unit. J Magn Reson Imaging 26(5):1328-1333

29. Dempsey MF, Condon B, Hadley DM (2001) Investigation of the factors responsible for burns during MRI. J Magn Reson Imaging 13(4):627-631

30. Buchli R, Boesiger P, Meier D (1988) Heating effects of metallic implants by MRI examinations. Magn Reson Med 7(3):255-261

31. Shellock FG, Shellock VJ (1998) Cardiovascular catheters and accessories: ex vivo testing of ferromagnetism, heating, and artifacts associated with MRI. J Magn Reson Imaging 8(6):1338-1342

32. Jezzard P, Clare S (1999) Sources of distortion in functional MRI data. Hum Brain Mapp 8(2-3):80-85

33. McRobbie DW (2006) MRI from picture to proton, 2nd edn. Cambridge University Press, Cambridge

34. Wadghiri YZ, Johnson G, Turnbull DH (2001) Sensitivity and performance time in MRI dephasing artifact reduction methods. Magn Reson Med 45(3):470-476

35. Franklin KM, Dale BM, Merkle EM (2008) Improvement in B1-inhomogeneity artifacts in the abdomen at 3T MR imaging 
using a radiofrequency cushion. J Magn Reson Imaging 27(6): $1443-1447$

36. Chinzei K, Yoshinaka K, Washio T (2006) Numerical simulations and lab tests for design of MR-compatible robots. In: The IEEE international conference on robotics and automation (ICRA), Orlando, FL, USA, pp 3819-3824

37. Diedrichsen J, Shadmehr R (2005) Detecting and adjusting for artifacts in fMRI time series data. Neuroimage 27(3):624-634

38. Yu N, Murr W, Blickenstorfer A, Kollias S, Riener R (2007) An fMRI compatible haptic interface with pneumatic actuation. In: The IEEE international conference on rehabilitation robotics (ICORR), Nordwijk, The Netherlands

39. Stoianovici D, Patriciu A, Petrisor D, Mazilu D, Kavoussi L (2007) A new type of motor: pneumatic step motor. IEEE/ASME Trans Mechatron 12(1):98-106

40. Gassert R, Moser R, Burdet E, Bleuler H (2006) MRI/fMRIcompatible robotic system with force feedback for interaction with human motion. IEEE/ASME Trans Mechatron 11(2):216-224

41. Yu N, Hollnagel C, Blickenstorfer A, Kollias SS, Riener R (2008) Comparison of MRI-compatible mechatronic systems with hydrodynamic and pneumatic actuation. IEEE/ASME Trans Mechatron 13(3):268-277

42. Chapuis D, Gassert R, Ganesh G, Burdet EABE, Bleuler HABH (2006) Investigation of a cable transmission for the actuation of MR compatible haptic interfaces. In: Gassert R (ed) The IEEE/RASEMBS international conference on biomedical robotics and biomechatronics (BioRob), pp 426-431

43. Chinzei K, Hata N, Jolesz FA, Kikinis RAKR (2000) Surgical assist robot for the active navigation in the intraoperative mri: hardware design issues. In: Hata N (ed) The IEEE/RSJ international conference on intelligent robots and systems (IROS), vol 1, pp 727-732

44. Larson BT, Erdman AG, Tsekos NV, Yacoub E, Tsekos PV, Koutlas IG (2004) Design of an MRI-compatible robotic stereotactic device for minimally invasive interventions in the breast. $\mathrm{J}$ Biomech Eng 126(4):458-465

45. Khanicheh A, Mintzopoulos D, Weinberg B, Tzika AA, Mavroidis C (2008) MR-CHIROD v.2: magnetic resonance compatible smart hand rehabilitation device for brain imaging. IEEE Trans Neural Syst Rehabil Eng 16(1):91-98
46. Hidler J, Hodics T, Xu B, Dobkin B, Cohen LG (2006) MR compatible force sensing system for real-time monitoring of wrist moments during fMRI testing. J Neurosci Methods 155(2):300 307

47. Gassert R (2006) MR-compatible robotics to investigate human motor control. $\mathrm{PhD}$ thesis

48. Kellman P, McVeigh ER (2005) Image reconstruction in SNR units: a general method for SNR measurement. Magn Reson Med 54(6):1439-1447

49. Stoianovici D (2005) Multi-imager compatible actuation principles in surgical robotics. Int J Med Robot Comput Assist Surg MRCAS 1(2):86-100

50. Chapuis D, Gassert R, Hartwig V, Vanello N, Bicchi A (2004) Prototype fMRI compatible actuators. Technical report, 30 May 2004

51. Vanello N, Hartwig V, Tesconi M, Ricciardi E, Tognetti A, Zupone G, Gassert R, Chapuis D, Sgambelluri N, Scilingo EP, Giovannetti G, Positano V, Santarelli MF, Bicchi A, Pietrini P, Rossi DD, Landini L (2008) Sensing glove for brain studies: design and assessment of its compatibility for fMRI with a robust test. IEEE/ASME Trans Mechatron 13(3):345-354

52. Mathworks MATLAB user's guide, 2008

53. Hutton C, Bork A, Josephs O, Deichmann R, Ashburner J, Turner $R$ (2002) Image distortion correction in fMRI: a quantitative evaluation. Neuroimage 16(1):217-240

54. Funai A, Fessler JA, Grissom W, Noll DC (2007) Regularized B1+ map estimation in MRI. In: The IEEE international symposium on biomedical imaging: macro to nano, vol 1-3, pp 616-619, 1383

55. Funai AK, Fessler JA, Yeo DTB, Olafsson VT, Noll DC (2008) Regularized field map estimation in MRI. IEEE Trans Med Imaging 27(10):1484-1494

56. Baselice F, Ferraioli G, Shabou A (2010) Field map reconstruction in magnetic resonance imaging using bayesian estimation. Sensors 10(1):266-279

57. McRobbie DW, Quest RA (2002) Effectiveness and relevance of MR acceptance testing: results of an 8 year audit. Br J Radiol 75(894):523-531

58. Koivula A (2002) Magnetic resonance image distortions due to artificial macroscopic objects-an example: correction of image distortion caused by an artificial hip prosthesis. $\mathrm{PhD}$ thesis 